and description of Pseudonocardiaceae fam. nov. Syst. Appl. Microbiol. 11:16-19.

5. Fendrich, C. 1988. Halovibrio variabilis gen. nov. sp. nov., Pseudomonas halophila sp. nov. and a new halophilic aerobic coccoid Eubacterium from Great Salt Lake, Utah, USA. Syst. Appl. Microbiol. 11:36-43.

6. Franzmann, P. D., E. Stackebrandt, K. Sanderson, J. K. Volkman, D. E. Cameron, P. L. Stevenson, T. A. McMeekin, and H. R. Burton. 1988. Halobacterium lacusprofundi sp. nov., a halophilic bacterium isolated from Deep Lake, Antarctica. Syst. Appl. Microbiol. 11:20-27.

7. Franzmann, P. D., U. Wehmeyer, and E. Stackebrandt. 1988. Halomonadaceae fam. nov., a new family of the class Proteobacteria to accommodate the genera Halomonas and Deleya. Syst. Appl. Microbiol. 11:16-19.

8. Hickman-Brenner, F., G. R. Fanning, M. J. Arduino, D. J. Brenner, and J. J. Farmer III. 1988. Aeromonas schubertii, a new mannitol-negative species found in human clinical specimens. J. Clin. Microbiol. 26:1561-1564.

9. Kompantseva, E. I. 1985. Rhodobacter euryhalinus sp. nov., a new halophilic purple bacterial species. Mikrobiologiya 54:974-982.

10. Rengpipat, S., T. A. Langworthy, and J. G. Zeikus. 1988. Halobacteroides acetoethylicus sp. nov., a new obligately anaerobic halophile isolated from deep subsurface hypersaline environments. Syst. Appl. Microbiol. 11:28-35.

11. Thacker, W. L., R. F. Benson, J. L. Staneck, S. R. Vincent, W. R. Mayberry, D. J. Brenner, and H. W. Wilkinson. 1988. Legionella cincinnatiensis sp. nov. isolated from a patient with pneumonia. J. Clin. Microbiol. 26:418-420.

12. Wilkinson, H. W., V. Drasar, W. L. Thacker, R. F. Benson, J. Schindler, B. Potuznikova, W. R. Mayberry, and D. J. Brenner. 1988. Legionella moravica sp. nov. and Legionella brunensis sp. nov. isolated from cooling-tower water. Antonie van Leeuwenhoek J. Microbiol. Serol. 139:393-402.

\title{
Errata
}

\section{List No. 20 (IJSB 36:354-356, 1986)}

Blastobacter aggregatus IFAM 1003, cited as DSM 111, should read DSM 1111. Blastobacter capsulatus IFAM 1004, cited as DSM 112, should read DSM 1112. Blastobacter denitrificans IFAM 1005, cited as DSM 113, should read DSM 1113.

\section{List No. 25 (IJSB 38:220-222, 1988)}

Methanogenicum olentangyi should read Methanogenium olentangyi; Methanothrix thermoacetophilia should read Methanothrix thermoacetophila. 